\title{
Gestión territorial: el tema pendiente en la planificación territorial en Honduras y Centroamérica
}

Claudia Nataly Mondragón Rivera

\section{Resumen}

En Honduras existen importantes avances vinculados al Ordenamiento y la Planificación Territorial, para compartir un caso, fue el primer país de Centroamérica en contar con una Ley de Ordenamiento Territorial (2003). Sin embargo, se cuentan a nivel nacional con 763 planes territoriales a escala municipal que poseen diferentes enfoques, de ellos, muy pocos han logrado tener una fase de implementación. Por lo que los resultados a nivel de sociedad y territorio no son tangibles. Casos similares se presentan en el resto de la Región Centroamericana. Por lo anterior es necesario generar los espacios, herramientas institucionales y técnicos municipales capacitados para fomentar la implementación de estos procesos, para garantizar una buena gobernanza del suelo, incidiendo de manera directa en la mejora de la calidad de vida de la población, como finalidad máxima del Ordenamiento Territorial.

Palabras Clave: Ordenamiento Territorial, Planificación, Gestión, implementación, Territorio, Municipio.

\section{Abstract}

In Honduras there are important advances linked to land use planning and planning, to share a case, was the first country in Central America to have a Law on Land Use Planning (2003). However, at the national level there are 763 territorial plans at municipal level that have different approaches, of which very few have managed to have an implementation phase. So the results at the level of society and territory are not tangible. Similar cases occur in the rest of the Region. Due to the above, it is considered that there is little progress in Honduras and Central America in territorial management processes and actions. Therefore, it is necessary to 
generate the spaces, institutional tools and municipal technicians trained to promote the implementation of these processes, to ensure good land governance, directly affecting the quality of life of the population, as a maximum objective Of the Territorial Ordering.

Keywords: Territorial Planning, Planning, Management, Implementation, Land, Municipality.

Claudia Nataly Mondragón, Honduras. (cn.mondragonrivera@gmail.com). Máster en Ordenamiento y Gestión del Territorio. Observatorio Universitario de Ordenamiento Territorial, Facultad de Ciencias Espaciales de la Universidad Nacional Autónoma de Honduras.

Fecha de Recepción: 6 de junio de 2017. Fecha de aprobación: 1 de octubre de 2017. 


\section{INTRODUCCIÓN}

\subsection{Territorio, Gestión y Gobernanza}

El sistema territorial es una construcción social inevitable que representa el estilo de desarrollo de una sociedad, se va formando desde la noche de los tiempos, y seguirá formándose inexorablemente hacia el futuro, mediante las actividades que la población practica sobre el medio físico y las interacciones que se producen entre ellas a través de los canales de relación que proporcionan funcionalidad al sistema. (Gómez Orea 2008).

Como en todo sistema, tres grandes elementos, conforman físicamente la sustancia del sistema territorial: la estructura, el funcionamiento y la imagen que transmite al exterior, a las que se añade una cuarta de índole temporal: la evolución hacia el futuro. (Gómez Orea 2008).

La estructura se manifiesta en las componentes del sistema, que son las siguientes:

- El medio físico o sistema natural en el estado actual (incluidos los usos primarios del suelo).

- La población, o conjunto de los ciudadanos.

- Las actividades humanas: de producción, de consumo y de relación social.

- El poblamiento o sistema de asentamientos de población.

- Los canales de relación a través de los que se intercambian personas, mercancías e información.

- Las instituciones y agentes que vertebran la sociedad.

- El marco legal que define las reglas del funcionamiento.

Por su parte el funcionamiento del territorio es aquel que se encuentra conformado por las relaciones entre lugares, generalmente polarizadas por una o más importantes, o por flujos de relación direccionales cual son las cuencas hidrográficas. El sistema territorial de un país se organiza en sub sistemas según niveles jerárquicos donde las unidades territoriales de cada nivel se integran en las del nivel superior. (Arévalo 2013).

A cada nivel corresponde estructura, potencialidades y problemas territoriales y específicos de rango de tal manera que no se pueden planificar desde niveles 
inferiores las estructuras que corresponden a niveles territoriales superiores ni prevenir o resolver sus problemas ni aprovechar sus potencialidades; pero ello no impide que las instituciones de niveles inferiores y todos los ciudadanos puedan participar en las decisiones que se adopten en niveles superiores. (Arévalo 2013).

La imagen territorial se refiere a lo que denominan base paisajista, que no es otra cosa, que los elementos exteriores que hacen ser llamativo al lugar, se hace referencia a lo visual en cuanto a las unidades de la dimensión territorial (Sánchez 2015).

La ordenación del territorio es la voluntad y la acción pública para mejorar la localización y disposición de los hechos en el espacio geográfico propio; especialmente de aquéllos a los que atribuimos un sentido estructurante o un mayor significado respecto a las necesidades y condiciones de vida de quienes lo habitan.

La voluntad y los actos para disponer, de la forma considerada más conveniente, determinados hechos en el territorio forman parte de las tareas habituales para su administración o gobierno. Se pueden encontrar fácilmente ejemplos, incluso correspondientes a situaciones muy poco evolucionadas, en los que el espacio geográfico atribuido a un órgano de poder ha sido considerado unitariamente para proponer determinadas acciones de ocupación y utilización. Actuaciones muy drásticas de ordenación o reordenación de conjunto se producen en situaciones extremas de dominio de espacios invadidos o colonizados. (Zoido Naranjo 1998).

Por otro lado, de acuerdo con el diccionario de Geografía aplicada y profesional, la gestión territorial es la implementación de un conjunto de acciones institucionales sobre el territorio, encaminadas a conseguir los objetivos emanados de una política de planificación en la que se señalan directrices para configurar un modelo territorial deseable. La praxis o intervención territorial demanda continuidad, una infraestructura institucional, recursos económicos y capacidades profesionales para la interlocución con diversos actores.

También involucra instrumentos de rendición de cuentas y evaluación de resultados. González (2011, p. 47) define la gestión territorial como "un conjunto de prácticas organizadas y ordenadas jerárquica y temporalmente, con sentido sistémico y dinámico, destinadas a intervenir en el ámbito territorial" y sugiere considerar "la dinámica y la variabilidad propia del territorio" e incorporar los atributos de "incertidumbre y certeza". El término gestión territorial remite a una de las fases convencionales de ordenación del territorio, antecedida del análisis territorial, del 
que se obtiene un diagnóstico y de una propuesta de planificación territorial, la cual se vale de instrumentos normativos y acuerdos sociopolíticos.

Y la gobernanza territorial se refiere a la capacidad de las sociedades de resolver sus asuntos y de contribuir al desarrollo de sus territorios mediante la articulación y participación de diversos actores territoriales, entre ellos (i) el Estado (gobernanza multinivel vertical), (ii) la sociedad civil, (iii) las agencias públicas localizadas territorialmente y (iv) el sector privado (gobernanza multinivel horizontal), en un contexto en el cual, el gobierno regional está enfocado en las oportunidades de los territorios, estimulado del crecimiento económico con inclusión social (RIMISP, 2010).

Este artículo se constituye como un análisis de la problemática existente a nivel regional, en donde se invierten tiempo y recursos en la planificación y no se da el paso a la gestión. Se constituye como un punto de partida en donde se desglosa la problemática y la realidad regional y se sientan las bases para una futura propuesta integral de soluciones a la temática.

\subsection{Mucha Planificación, Poca Gestión, Deficiente Gobernanza- Centroamérica una Región de Retos}

A pesar que en la región existe una importante experiencia, habiendo países como El Salvador, que ha desarrollado todos los planes a nivel nacional, regional y subregional, o como Honduras en donde se ha desarrollado planificación a nivel regional y municipal, los planes no se ejecutan, y mucho menos se instrumentalizan al nivel municipal.

En la mayoría de los casos los planes no se aplican, quedando archivados 0 engavetados en anaqueles de políticos y/o técnicos; entre las causas que provocan que los instrumentos no se ejecutan a nivel local se encuentran:

- Falta de capacidades municipales; en la mayoría de las municipalidades no se cuenta con recursos económicos ni humanos con conocimiento en el tema.

- Falta de capacitación de los técnicos; en algunas municipalidades existen recursos humanos potenciales, pero los mismos no poseen la formación y/o la experiencia; además en los procesos o no se les capacita o la capacitación resulta insuficiente o deficiente. 
- Falta de interés en las autoridades locales, que se ven más motivados por pequeñas obras y proyectos que "generan más votos" que por este tipo de instrumentos con visión de medio y largo plazo.

- Inexistencia de normativas locales, y falta de leyes nacionales que faculten claramente a los municipios a la regulación de los usos del suelo, en especial, sobre los usos urbanísticos.

Existen a nivel nacional en Honduras, 763 planes territoriales a escala municipal que poseen diferentes enfoques, sin embargo, muy pocos han logrado tener una fase de implementación y gestión, por lo que los resultados a nivel de sociedad y territorio no son tangibles. Por lo anterior es necesario generar los espacios y herramientas institucionales y técnicos para fomentar la implementación de estos procesos, para garantizar una buena gobernanza del suelo (Ver Figura 1).
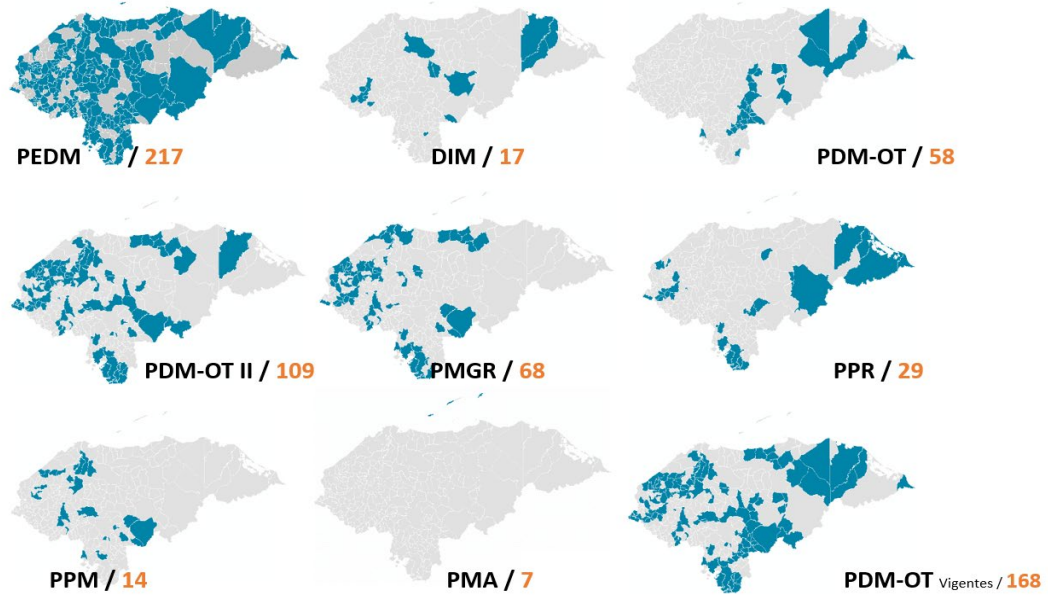

Simbología de la Figura.

PEDM Plan Estratégico de Desarrollo Municipal

DIM Diagnóstico Integrado Municipal

PDM - OT Plan de Desarrollo Municipal con Enfoque de Ordenamiento

Territorial

PDM-OT Plan de Desarrollo Municipal con Enfoque de Ordenamiento

Territorial, segunda metodología

PMGR Plan Municipal de Gestión de Riesgos

PPR Plan de Prevención de Riesgos

PPM Plan de Prevención Mitigación Municipal

PMA Plan Municipal Ambiental

PDM-OT Plan de Desarrollo Municipal con Enfoque de Ordenamiento Territorial Vigentes

Figura 1. Planes Territoriales a escala municipal existentes en Honduras. 
No existen en la Región casos significativos de buenos procesos de implementación de planificación territorial, por lo que la investigación se posiciona como una novedad y una oportunidad para los gobiernos locales en comenzar a gestionar los procesos de manera participativa y de potenciar las capacidades locales mediante el uso de las herramientas propuestas.

\section{Resultados obtenidos de la poca implementación de los instrumentos de OT}

\subsection{Escasez de algunos datos básicos: cartografía y falta de datos históricos}

Uno de los problemas que mayor incide en la debilidad de los planes de ordenamiento territorial en los países centroamericanos, es el déficit de información cartográfica y de algunas variables territoriales e indicadores (Demanda Biológica de Oxígeno DBO, Demanda Química de Oxígeno DBQ, deforestación etc.); si bien en los últimos años se ha incrementado la toma y análisis de datos de estudios y proyectos, financiados por los gobiernos o la cooperación internacional y multilateral (Banco Interamericano de Desarrollo BID, Banco Mundial BM, Banco Centroamericano de Integración Económica BCIE, Unión Europea UE, Agencia Andaluza de Cooperación Internacional para el Desatollo AACID, Agencia Alemana de Cooperación Técnica GIZ etc.), todavía existe escasez de datos, sobre todo al nivel regional y municipal; además no existen datos históricos, por lo que se dificulta la fase de prospectiva, al desconocer la evolución de algunos indicadores. Todo ello incide en cierta debilidad de los planes, que se suele suplir, mediante análisis participativo y algunos métodos de medición cualitativos.

Esto es importante ya que los SIG ofrecen una gran variedad de utilidades y aplicaciones relacionadas con los trabajos específicos de ordenamiento urbano y territorial. Por ejemplo, contribuyen en: (Fernández 2011).

- las tareas de almacenamiento y sistematización de la información de entes públicos y privados (censos, catastro, bases inmobiliarias, patrimonio público, padrones industriales y comerciales, redes de infraestructura urbana, etc.);

- la identificación, cuantificación y análisis de la distribución espacial de cualquier fenómeno urbanos o de carácter territorial;

- el análisis de tendencias espaciales para la definición de lineamientos territoriales;

- la evaluación de modificaciones de normas urbanas y trabajos de prospectiva territorial; 
- los diagnósticos de situación y el diseño de políticas territoriales diversas, desarrollo de planes de sector, planes parciales, códigos urbanos, entre otras;

- el control y la gestión de la información para los procesos de toma de decisión, por ejemplo, el seguimiento georreferenciado de expedientes.

\subsection{Sobre la falta de una visión regional de ordenamiento territorial}

A pesar que se han realizado algunos intentos de armonización y cohesión de las políticas territoriales, en la actualidad no existe una visión centroamericana; además las tensiones políticas recientes han incidido negativamente en el proceso de integración centroamericana, marcada por algunos eventos: a) Golpe de estado de Honduras y crisis política hondureña. b) situación de Nicaragua con unas reformas constitucionales polémicas, c) tensiones fronterizas entre Costa Rica y Nicaragua en el río San Juan, y d) salida de Panamá del Parlamento Centroamericano, entre otras.

No obstante, para el desarrollo territorial del área centroamericana es fundamental una visión supranacional, ante un mundo globalizado que se organiza y se defiende mediante grandes regiones económicas (Unión Europea UE, Estados Unidos EEUU, Mercado Común del Sur MERCOSUR, Alianza Bolivariana para los Pueblos de Nuestra América ALBA etc.), máxime para estos países, de pequeño tamaño, que apenas llegan a los 100 mil Km2, donde la historia, cultura, vínculos sociales y económicos, son comunes.

También cabe señalar que se han realizado algunos esfuerzos sectoriales, como el Plan Puebla-Panamá, con enfoque de desarrollo económico a lo largo de la carretera panamericana, o el Corredor Biológico Mesoamericano, para la interconexión de áreas protegidas; pero los mismos hasta la fecha han resultado infructuosos y estériles.

Los proyectos ejecutados al nivel regional, con temas integrales y sectoriales, vinculados al OT; por citar algunos de ellos:

- A nivel de Mesoamérica, el Plan Puebla Panamá (carretera interoceánica logística e interconexión eléctrica centroamericana) y el Corredor Biológico Mesoamericano. 
- Al nivel de Centroamérica: SIECA, CCJ, PARLACEN, entre otros.

- Al nivel binacional. El Tratado de Libre Comercio (TLC), el proyecto trinacional de la cuenca del río Lempa etc.

\subsection{Sobre algunas confusiones y errores metodológicos}

\section{- Diferencias de ordenamiento territorial y urbanismo}

No existe una clara separación entre los anteriores, por lo que sucede a menudo una gran confusión en los términos y en su aplicación.

Es importante acotar que la diferencia entre ambos radica principalmente en el espacio geográfico a analizar, mientras el primero ordena los territorios de municipios, regiones o naciones a través de una plataforma o sustento normativo que permita regular las actuaciones en el suelo, el urbanismo se centra en el aspecto funcional y espacial de la ciudad en términos de los requerimientos de la vida humana.

\section{- Desconocimiento y falta de desarrollo de la fase de prospectiva}

De la experiencia en el desarrollo de estudios de ordenamiento territorial, y la lectura de informes, libros y otros, relacionados con la materia, se considera que es la fase más compleja y peor desarrollada en la región.

Aún considerando que existen diferentes planteamientos metodológicos, casi todos los autores relacionados con el tema desarrollan tres grandes fases: diagnóstico 0 inicial, prospectiva o intermedia, y propuestas o final (también planificación 0 programación por otros); luego estas se subdividen en otras, y se plantean diferentes herramientas.

En la fase de diagnóstico existe mucha literatura, presentando coincidencias en cuanto alcances, objetivos y herramientas; en la fase de propuestas, aun existiendo obviamente mayores diferencias, también hay mucho desarrollado, y de buena calidad; sin embargo, la fase de prospectiva, o no se desarrolla (en Latinoamérica en muchos de los casos), o se desarrolla mal; existiendo gran diferencia en los planteamientos, graves errores en los conceptos, y un bajísimo nivel, si lo comparamos con otras etapas de los estudios; lo anterior obedece a cuestiones 
obvias, es más sencillo determinar el estado actual que el futuro; lo cual no quita, que es probablemente la etapa más importante en un estudio de ordenamiento territorial, constituyéndose en el alma de un plan; si no sabemos hacia donde queremos ir ¿Cómo lo haremos?; es por ello que gran parte de los estudios son incompletos o tienen algunos fallos en su concepción, ya que no parten de un buen análisis prospectivo.

La prospectiva o pronóstico, constituye una fase intermedia en la elaboración de un plan de ordenamiento territorial, entre el diagnóstico y las propuestas (planificación o programación), y se refiere a la predicción del futuro. (Gómez Orea 2010).

Como señalaba Gastón Berger (1959, p. 270-275), antes que una disciplina o un método, la prospectiva es una actitud, que se basa en cinco necesidades:

1. Mirar lejos: la actitud prospectiva nos hace mirar hacia lo lejos

2. Mirar con amplitud: en los asuntos humanos;

3. Analizar en profundidad

4. Arriesgarse

5. Pensar en el individuo: desde muchos puntos de vista.

Por su parte Michel Godet, se basa en el humanismo para proponer que el futuro puede ser creado y modificado por las acciones de los actores sociales, ya sea individuales u organizados, y propone estudios que caractericen la sociedad futura en sus diversos enfoques: social, económico y cultural.

La prospectiva, sea cual sea, constituye una anticipación (preactiva y proactiva) para iluminar las acciones presentes con la luz de los futuros posibles y deseables. Prepararse ante los cambios previstos no impide reaccionar para provocar los cambios deseados. Dentro de la lógica del triángulo griego, el color azul de la anticipación sólo puede transformarse en el verde de la acción con el amarillo de la adaptación de los actores implicados. Godet, 2004.

\section{- Confusión entre ordenamiento territorial y catastro (y registro de la propiedad)}

En algunos países como Honduras existe algún desconcierto ya que se confunde ordenamiento territorial con catastro y registro de la propiedad. 


\section{- $\quad$ Otras. Realismo mágico}

A lo largo de la experiencia en talleres participativos de procesos territoriales, se concluye que existe una gran confusión al nivel local, ya que los actores no entienden la utilidad del instrumento; en ocasiones se suele asimilar a una mera planificación económica, y en otras, se considera un instrumento todopoderoso y omnipresente que lo resuelve todo.

\subsection{La regionalización. Dilema de las unidades definidas para la planificación}

Otro de los grandes retos y dilemas es sobre las unidades de planificación sobre los cuales se debe desarrollar los procesos territoriales; por un lado existe el enfoque por unidades político-administrativas y de otro las cuencas hidrográficas, existiendo modelos mixtos según conveniencia.

Mas allá de las bondades de un sistema u otro, aunque en lo personal considero más eficaz el modelo por unidades político-administrativas, por la historia, funcionalidad socioeconómica, identidad, cultura y pequeños avances en la materia, dejando las cuencas para la planificación del recurso agua, lo importante es que el país apueste a un sistema único y permanente, es decir, que se mantenga inviolable en el tiempo.

Sin embargo, en la mayoría de los países, se divaga de un lado a otro, en función de los vaivenes políticos y/o modas, de tal manera que ahora se planifica por cuencas y más tarde por departamentos, para volver de nuevo a las cuencas; como casos paradigmáticos negativos nos encontramos El Salvador, que después de 10 años planificando su territorio sobre unidades mixtas basadas en las cuencas y haber finalizado todos los planes regionales y subregionales, parece haber decidido regresar a los departamentos, y Honduras, que después de años basándose en el departamento (La Ley original así lo planteaba) como unidad básica de planificación territorial regional, ha creado una nueva Ley de Visión de País, Plan de Nación, donde se organiza el país por cuencas hidrográficas. 


\subsection{Dilema de la democracia o tecnocracia para el desarrollo de los instrumentos de planificación territorial. Instrumentos débiles o inaplicables como alternativas al desarrollo}

Otra de las cuestiones que inciden en la falta de aplicación de los planes y baja incidencia de los mismos son el dilema entre la tecnocracia, planes muy técnicos pero de escasa aceptación y compresión social, o democráticos, muy participativos, pero excesivamente simples y de escaso interés, convirtiéndose en una mera aglomeración de proyectos de inversión.

Realización de instrumentos en ocasiones muy complejos, con lo cual no se entienden en el nivel local, y por ende no se aplican, y en otras ocasiones excesivamente simples y livianos, con bajo contenido en ordenamiento territorial; se fundamentan en un banco de proyectos de inversión sin una zonificación y normas de regulación de usos del suelo; algo llamado planes estratégicos.

En mi opinión, los procesos participativos son muy interesantes, pero deben estar acotados por la parte técnica, ya que los estudios de ordenamiento territorial tienen una componente científico-técnica que no puede ser obviada (Ej. No se puede recomendar la construcción en áreas de amenazas naturales graves); para ello se deben realizar estudios técnicos profundos, y extraer las conclusiones de una manera sinóptica, para posteriormente acudir a procesos participativos sencillos, fáciles y comprensibles.

Según Godet 2004, aparecen dos errores simétricos a evitar. El primero consiste en pensar en voz alta con los expertos al servicio de la acción, olvidando la adaptación. No es una buena idea querer imponer una buena idea. El segundo consiste en desechar a los expertos y la materia azul de las evaluaciones para dar la palabra al pueblo y otorgar privilegios a la materia amarilla de los consensos actuales. Sin prospectiva cognitiva, la prospectiva participativa se vuelve vacía y gira en redondo hacia el presente. El ideal de consenso de las actuales generaciones es muchas veces un acuerdo momentáneo para que nada cambie y para transmitir a las futuras generaciones la carga de nuestras irresponsabilidades colectivas. Una prospectiva como la descrita, aunque sea participativa, es contraria a la definición misma del desarrollo sostenible. Consagra el triunfo de los egoísmos individuales a 
corto plazo (solo son injustas las desigualdades de las que no nos aprovechamos) en detrimento de los intereses colectivos a largo plazo. Las decisiones valientes a afrontar en el futuro son raramente consensuadas, y si la prospectiva debe ser participativa, la estrategia en la que se inspira vuelve a los representantes electos, y estos deben ofrecer acto de voluntad y de valentía para evitar la trampa y la demagogia participativa.

\subsection{Sobre la presión del sector privado y desconocimiento ciudadano. El efecto riqueza individual y pobreza colectiva}

La planificación territorial tiene indefectiblemente una afectación al uso del suelo, algo que los privados lo sienten como una amenaza (En la región impera el lema "esta es mi tierra y en ella puedo hacer lo que quiera"), de esta manera el interés público debe primar sobre los intereses de los privados, algo que en la mayoría de los casos no es aceptado; ello hace que las empresas y particulares (en especial para el derecho de urbanización y construcción) no quieran sujetarse a normas de regulación, por tanto se provoca que:

- Hay grandes intereses comprometidos

- La defensa del interés común está bajo la presión de intereses particulares: riesgo de corrupción, evasión, ausencia de control etc.

- Falta de transparencia de la información, descontrol

- Baja aplicación (o no aplicación de los planes)

Producto de lo anterior las leyes de ordenamiento territorial y urbanismo son capadas, y se convierten en discursos filosóficos sobre conceptos, instrumentos, alcances y metodología, pero en ninguno de los casos aterriza al nivel local; en la mayoría de países a excepción de Costa Rica, y en cierta medida El Salvador que ha avanzado en la materia, no existe el concepto legal de suelo no urbanizable, a excepción de las áreas protegidas; aunque infelizmente también se urbanizan en algunos casos.

\subsection{Sobre la discontinuidad e intermitencia de las políticas públicas}

Otro de los problemas que afronta la planificación territorial es la miopía política y caciquismo; algo que afecta enormemente al nivel estatal y en menor medida 
al nivel local, ya que el primero indefectiblemente en la mayoría de los casos (a excepción de Nicaragua que ha reformado la constitución y en Honduras que también es permitido) no se permite la reelección, y en el segundo si, existiendo alcaldes, regidores y líderes que se mantienen por varios periodos; no obstante los continuos cambios de gobierno, suponen cambios drásticos en las políticas públicas, de tal manera que el plan que diseñó o desarrolló el gobierno anterior es visto por el entrante como algo negativo, de esta manera se deroga o engaveta, y se establecen otras políticas territoriales, en muchos casos antagónicas u opuestas a las originales.

Lo anterior ha generado problemas territoriales que agravan la realidad social de comunidades rurales y urbanas, acrecentando conflictos relacionados con:

- Desequilibrio territorial, que según el Diccionario de Ordenación del Territorio de la Universidad de Alicante se define como Desigualdades sociales inaceptables entre áreas con significación propia de una misma entidad política unitaria.

- Degradaciones ecológicas y despilfarro de los recursos naturales,

- Ignorancia de los riesgos en la localización de actividades y usos del suelo

- Mezcla de usos del suelo, y conflictos entre actividades y sectores

- Dificultades territoriales para dotar de equipamientos y servicios públicos

- Descoordinación de los organismos públicos y los organismos financieros y cooperantes.

- Poca motivación para la inversión privada en proyectos de desarrollo: agrícolas, turísticos etc.

\section{Pinceladas De Una Posible Solución}

Los conceptos de Gestión territorial y Desarrollo Local están estrechamente relacionados, Barreriro (2000) plantea una definición muy operativa de desarrollo local, entendiéndolo como "el resultado de una acción de los actores o agentes que inciden (con sus decisiones) en el desarrollo de un territorio determinado. Estas decisiones no solamente se toman a una escala local, sino que existen decisiones que tomadas en otra escala (nivel nacional e internacional) tienen incidencia en el desarrollo de un territorio dado. La preeminencia de las decisiones de los actores locales, por sobre otras decisiones que no responden a los intereses locales, es 
lo que define un proceso de desarrollo local". Es importante determinar entonces mediante procesos participativos, cuáles son los intereses locales para cada territorio.

Según Gonzáles Fuenzalida (2011), la gestión territorial es la acción de ingeniería, ya que requiere instrumentos para su diseño y ejecución. En ese sentido, la planificación es un aspecto fundamental de toda acción que se lleve a cabo en el territorio, pero es primordial señalar que tanto el método de planificación, como el modo de entender los procesos derivados, son aspectos relevantes que pueden determinar que la generación de un plan llegue o no a ser un obstáculo para la obtención de los fines propuestos o incluso, que se convierta en una traba a los propios propósitos.

Será necesario entonces generar un método, tomando en cuenta la escala y la realidad regional, que permita generar los espacios necesarios para la correcta implementación de los Planes de OT, incidiendo de manera directa en la calidad de vida de la población.

Una posible solución ante la problemática es la conformación de un ente gestor para la implementación del PMOT. Se describen a continuación los factores y procedimientos necesarios para conformar un ente rector con la finalidad de gestionar el plan.

Se propone a través de esta sugerencia la conformación de un ente gestor de los planes municipales de Ordenamiento Territorial, con la finalidad de dirigir el cumplimiento de los productos de esta fase y la futura implementación de la propuesta general de esta Tesis, el cual debe ser coordinado y dirigido por la estructura de la Mancomunidad. 
Las funciones del ente gestor deben ser las siguientes:

\begin{tabular}{|l|}
\hline \multicolumn{1}{|c|}{ FUNCIONES ENTE GESTOR } \\
\hline $\begin{array}{l}\text { Animación, de los agentes públicos y privados para que materialicen las propuestas que el plan } \\
\text { les haya asignado. }\end{array}$ \\
\hline $\begin{array}{l}\text { Control, velando para que todas las medidas, tanto normativas como de inversión, se realicen } \\
\text { dentro de las previsiones del plan y se ajusten a derecho. }\end{array}$ \\
\hline $\begin{array}{l}\text { Coordinación, de las actuaciones de todos los agentes públicos y privados, dentro de las } \\
\text { previsiones del plan, de forma coherente con las actuaciones de otro origen que se desarrollen en } \\
\text { su ámbito territorial. Por otro lado, coordinará las acciones de promoción y difusión realizadas } \\
\text { por las administraciones que intervengan en el plan. }\end{array}$ \\
\hline $\begin{array}{l}\text { Seguimiento, del estado de materialización de cada una de las fases del plan y de cada una de las } \\
\text { actuaciones que lo conforman. Para ello se tomarán en cuenta indicadores fisicos y financieros, } \\
\text { ajustados al carácter especifico de cada determinación y conforme a lo estipulado en el propio } \\
\text { plan. En función de ello se propondrán las modificaciones y adaptaciones que se consideren } \\
\text { pertinentes. }\end{array}$ \\
\hline $\begin{array}{l}\text { Evaluación, identificación, estimación y valoración de los efectos del plan en todos los aspectos } \\
\text { relevantes y sectores. }\end{array}$ \\
\hline $\begin{array}{l}\text { Información: elaborar informes sobre su actividad en relación con todas las funciones enunciadas } \\
\text { y remitirlos a las autoridades responsables. }\end{array}$ \\
\hline
\end{tabular}

Fuente: Domingo Gómez Orea. 2010.

El esquema organizativo del ente gestor, coordinado políticamente y técnicamente por la mancomunidad se propone sea el siguiente: 


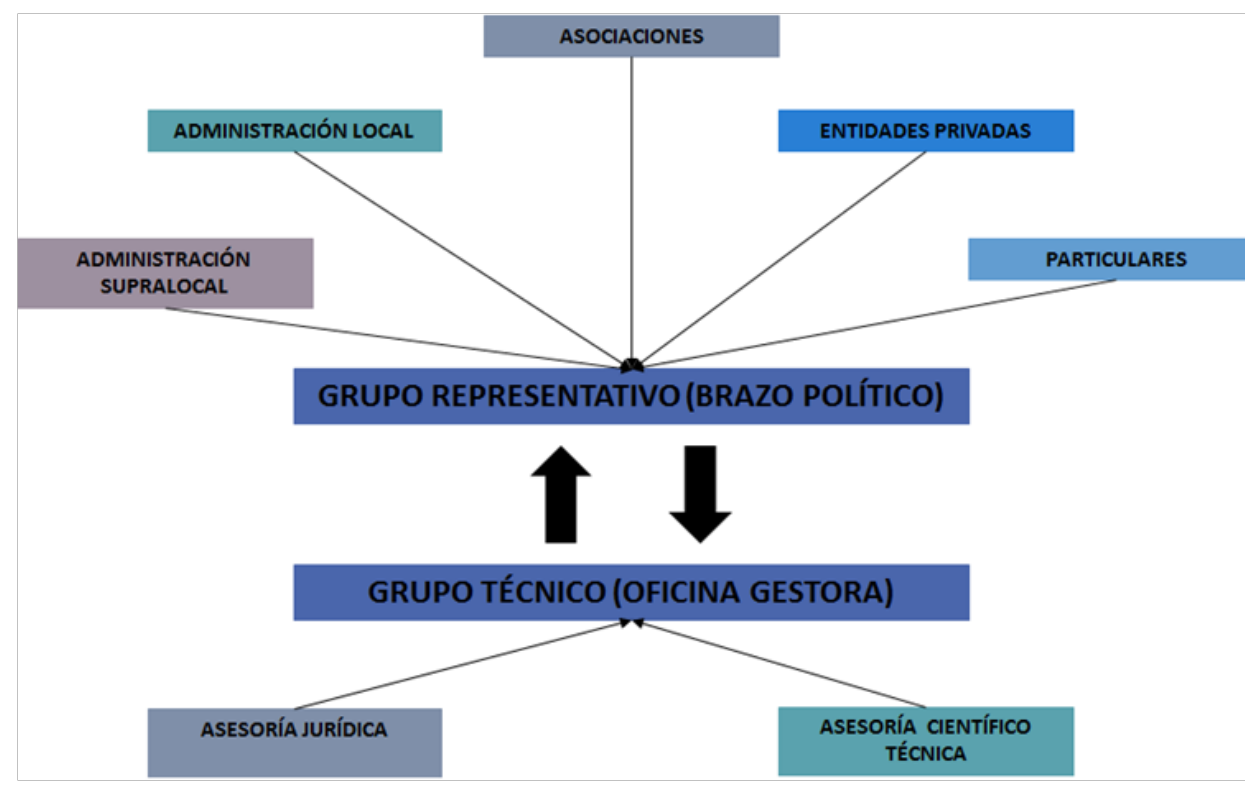

Figura 2. Esquema Organizativo del Ente Gestor Fuente: Domingo Gómez Orea. 2010.

Las funciones específicas de los grupos que conformarán el ente gestor son las siguientes:

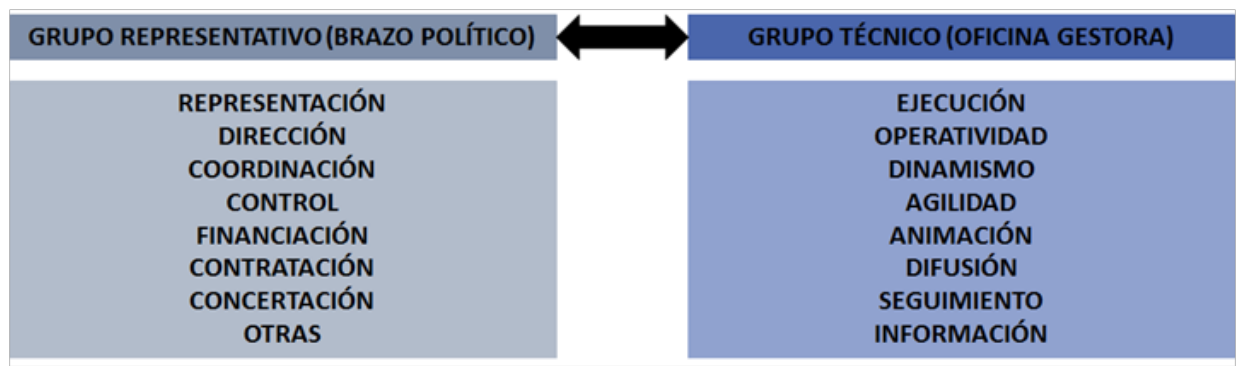

Figura 3. Funciones del Ente Gestor según Grupo Fuente: Domingo Gómez Orea. 2010. 


\section{CONCLUSIONES}

- A pesar que en la región existe una importante experiencia, habiendo países como El Salvador, que ha desarrollado todos los planes a nivel nacional, regional y subregional, o como Honduras en donde se ha desarrollado planificación a nivel regional y municipal, los planes no se ejecutan, y mucho menos se instrumentalizan al nivel municipal. En la mayoría de los casos los planes no se aplican, quedando archivados o engavetados en anaqueles de políticos y/o técnicos; entre las causas que provocan que los instrumentos no se ejecutan a nivel local.

- No existen en la Región casos significativos de buenos procesos de implementación de planificación territorial, por lo que la investigación se posiciona como una novedad y una oportunidad para los gobiernos locales en comenzar a gestionar los procesos de manera participativa y de potenciar las capacidades locales mediante el uso de las herramientas propuestas.

- Uno de los problemas que mayor incide en la debilidad de los planes de ordenamiento territorial en los países centroamericanos, es el déficit de información cartográfica y de algunas variables territoriales e indicadores.

- A pesar que se han realizado algunos intentos de armonización y cohesión de las políticas territoriales, en la actualidad no existe una visión centroamericana; además las tensiones políticas recientes han incidido negativamente en el proceso de integración centroamericana, marcada por algunos eventos de carácter internacional.

- Otra de las cuestiones que inciden en la falta de aplicación de los planes y baja incidencia de los mismos son el dilema entre la tecnocracia, planes muy técnicos, pero de escasa aceptación y compresión social, o democráticos, muy participativos, pero excesivamente simples y de escaso interés, convirtiéndose en una mera aglomeración de proyectos de inversión.

\section{BIBLIOGRAFÍA}

- Arévalo, José Antonio. (2013). El Sistema Territorial, Modelo Territorial y Evolución Tendencial del Sistema Territorial. Universidad Nacional Federico Villareal. Perú 
- Barreiro, Fernando. 2000. Desarrollo desde el Territorio: a propósito del desarrollo local. Madrid, España. en En http://www.iigov.org

- Berger, Gaston. (1964). Phenomenologié du temps et prospective. Paris, P.U.R. p.275.

- Centro Latinoamericano para el Desarrollo Rural. (2010). Territorios Rurales en Movimiento. Documento de Trabajo No. 110. Santiago, Chile.

- Fernández, Silvina Edith. (2011). Sistemas de Información Geográfica para el Ordenamiento Territorial. La Provincia de Buenos Aires, Argentina.

- Godet, Michael. (2007). Prospectiva Estratégica. Problemas y métodos. Paris: Cuadernos de LIPSOR.

- Gómez Orea, Domingo (2008), Ordenación del territorio. Instituto Tecnológico Geominero de España/Editorial Agrícola España: España.

- Gonzáles Fuenzalida, Luis. 2011. Gestión del Territorio, un método para la intervención territorial. Santiago de Chile, Universidad de Chile. Disponible en: http://repositorio.uchile.cl/bitstream/handle/2250/120283/Gonzalez_Luis_ Gestion_territorio.pdf?sequence=1\&isAllowed=y

- Lopez Trigal, Lorenzo. (2015). DICCIONARIO DE GEOGRAFÍA APLICADA Y PROFESIONAL Terminología de análisis, planificación y gestión del territorio. España, Universidad de León.

- Sánchez Peña, Leonor. (2015). Imagen del Sistema Territorial y su Percepción. Nicaragua.

- Universidad de Alicante. Laboratorio de Climatología (2014). Diccionario de Ordenación Territorial. España.

- Zoido Naranjo, Florencio (1998). Geografía y Ordenación del Territorio. Barcelona, España. Nuevas fronteras de los contenidos geográficos, p. 19-31 